\title{
Conservative Chaos Generators with CCII+ Based on Mathematical Model of Nonlinear Oscillator
}

\author{
Jiř́ PETRŽELA, Josef SLEZÁK \\ Dept. of Radio Electronics, Brno University of Technology, Purkyňova 118, 61200 Brno, Czech Republic \\ petrzelj@feec.vutbr.cz
}

\begin{abstract}
In this detailed paper, several novel oscillator's configurations which consist only of five positive second generation current conveyors (CCII+) are presented and experimentally verified. Each network is able to generate the conservative chaotic attractors with the certain degree of the structural stability. It represents a class of the autonomous deterministic dynamical systems with two-segment piecewise linear (PWL) vector fields suitable also for the theoretical analysis. Route to chaos can be traced and observed by a simple change of the external dc voltage. Advantages and other possible improvements are briefly discussed in the text.
\end{abstract}

\section{Keywords}

Analog oscillator, attractor, conservative dynamics, chaos, state space.

\section{Introduction}

A huge pile of the journal articles solving problems with chaos and other types of complex behavior have been published from its early discovery about forty years ago. The reliable parts of these publications are about methods how to create chaotic waveform by an oscillator. With the latest progress in the analog integrated circuit design the new electronic devices are developed and thus used inside various applications including chaos generators. It is also the case of CCII+ which is a modern functional block with three ports capable to work at high frequencies.

For any circuit structure and purpose, having certain knowledge about the basic properties of the generated waveforms in time and frequency domain is essential. Understanding a complex dynamics is useful also from the theoretical standpoint. Chaos can be considered as the universal phenomenon due to the normalization of system parameters and arbitrary interpretation of state variables. That is the reason why chaos has been experimentally confirmed and reported in many distinct scientific fields, for example in power electronics, digital circuits, ecology, chemical reactions, population growth, optics, etc.

The overall information about conservative dynamics can be found in [1]. Several conservative systems taken over from the technical practice are also provided in this publication. The very simple example of driven dynamical system is mentioned in [2] where PWL type nonlinearity and periodic driving force is assumed. The integrator based conservative chaotic oscillator with two modifications is presented and verified in [3]. Both presented systems model dynamics of the Nosé-Hoover's thermostat.

This paper is organized as follows. Section two gives the necessary mathematical background together with the numerical integration of the studied systems. The third section is focused on the individual circuits. They are synthesized in order to obtain different structures with qualitatively equivalent conservative dynamics. The next section is aimed on the experimental verification of final oscillators.

\section{Numerical Analysis}

Let us consider a mathematical model of the nonlinear oscillator with two-segment PWL vector field

$$
a_{3} \dddot{x}+a_{2} \ddot{x}+a_{1} \dot{x}+a_{0} x=b_{0} \max (x-\wp, 0)+\mathfrak{I}
$$

where dots denote the time derivatives, $\wp$ is the breakpoint and $\mathfrak{I}$ represents the offset of the nonlinear function. Note that we can assume $a_{3}=1$ without a loss of generality. Straight-forward analysis of this differential equation following the rules of linear algebra leads to the three possibilities for the number of fixed points. There are no equilibria if $\mathfrak{I}>\wp$ and one fixed point located on the boundary plane occurs when $\mathfrak{I}=\wp$. We get two equilibria per region only in the particular case $\mathfrak{I}<\wp$ and they are in positions $\mathbf{x}_{0}=\left[\begin{array}{lll}\mathfrak{I} / a_{0} & 0 & 0\end{array}\right]^{\mathrm{T}}$ and $\mathbf{x}_{0}=\left[\left(b_{0} \wp-\mathfrak{I}\right) /\left(b_{0}-a_{0}\right) \quad 0 \quad 0\right]^{\mathrm{T}}$, respectively. Only the latter case is important from the viewpoint of the conservative chaos generation. It is not hard to obtain the characteristic equation for the left segment of the vector field in the form

$$
x<\wp \Rightarrow \lambda^{3}+a_{2} \lambda^{2}+a_{1} \lambda+a_{0}=0,
$$

and similarly for the right segment

$$
x>\wp \Rightarrow \lambda^{3}+a_{2} \lambda^{2}+a_{1} \lambda+a_{0}-b_{0}=0 .
$$

It is well known that the eigenvalues are roots of these polynomials and determine the local behavior of the system 
in the neighborhood of the fixed points. The eigenvalues can be computed symbolically by means of the Cardan formulas. This kind of approach can give us an idea when eigenvalues are complex conjugated forming a composition of the eigenplane and eigenvector or if the geometry is more likely a combination of three eigenvectors. Also the question about the stability indexes can be easily answered. In spite of this, the existence of chaos will remain uncovered. Due to this, it is time to leave the symbols and work with numbers. The basic requirement for no dissipation tell us that $a_{2}=0$. Other values are given in the publication [4]

$$
a_{1}=2.7, \quad a_{0}=1, \quad b_{0}=2,
$$

or can be obtained using the stochastic optimization way described in [5]

$$
a_{1}=8.1, \quad a_{0}=5.5, \quad b_{0}=8.7 .
$$

In both cases the parameters of the nonlinear function were the same, in detail $\mathfrak{I}=-1$ and $\wp=0$. The set of parameters (4) leads to the following real and a pair of complex conjugated eigenvalues

$$
\begin{aligned}
& x<\wp \quad \Rightarrow \quad \lambda_{1,2}=0.177 \pm 1.672 j \quad \lambda_{3}=-0.354 \\
& x>\wp \Rightarrow \lambda_{1,2}=-0.177 \pm 1.672 j \quad \lambda_{3}=0.354
\end{aligned}
$$

The same geometry of the vector field holds for the second case (5) as expected, i.e.

$$
\begin{aligned}
& x<\wp \Rightarrow \lambda_{1,2}=0.323 \pm 2.9 j \quad \lambda_{3}=-0.646 \\
& x>\wp \Rightarrow \lambda_{1,2}=-0.194 \pm 2.866 j \quad \lambda_{3}=0.388
\end{aligned}
$$

The geometry of the vector field is

$$
x<\wp \Rightarrow \Re_{\mathrm{u}}^{2} \oplus \Re_{\mathrm{s}}^{1} \quad x>\wp \Rightarrow \mathfrak{R}_{\mathrm{u}}^{1} \oplus \mathfrak{R}_{\mathrm{s}}^{2} .
$$

The numerical integration using the fourth-order RungeKutta method with final time $t_{\max }=400$, time step $t_{\text {step }}=0.04$ and zero initial condition is shown in Fig. 1 for the first set of parameters (4) with the state variables ranges $x \in(-1,1)$, $y \in(-2,2), z \in(-3,3)$. Analogically, Fig. 2 corresponds to the attractor when (5) is set up. In this case, the axes are fixed at $x \in(-3,3), y \in(-5,5), z \in(-10,10)$. Note that such ranges of the individual state variables are narrow enough for the practical implementation. This is indeed important because of current and/or voltage saturation of the active devices. The numerical analysis involving the computation of the spectrum of Lyapunov exponents as well as the dimensions of the state space attractors reveals that the chaotic regions are significantly surrounded by the regions with unbounded solution. The system parameters $\mathfrak{I}$ and $\wp$ do not affect the eigenvalues so it can be hardly considered as a natural bifurcation parameters. Also a thin basin of attraction can cause serious problems, especially in case of experimental verification. Chaos is often destructed simply by switching on/off the power supply.

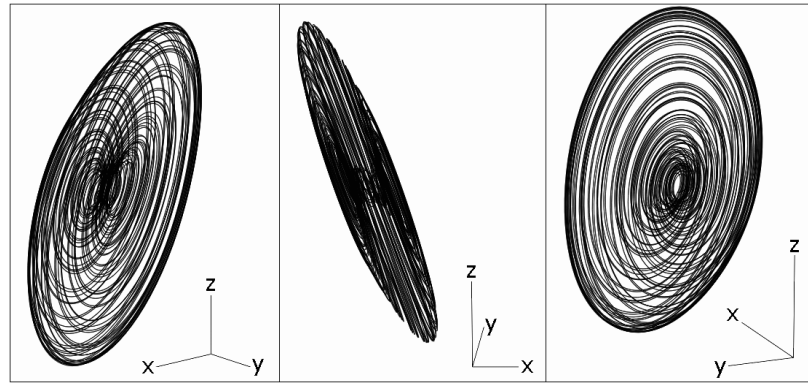

Fig. 1. 3D state space trajectories for the $1^{\text {st }}$ set of parameters.

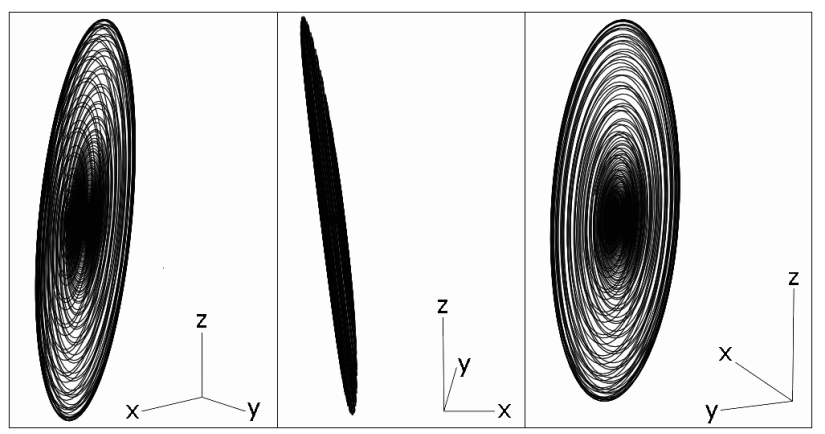

Fig. 2. 3D state space trajectories for the $2^{\text {nd }}$ set of parameters.

\section{Circuit Realization}

Before speaking about particular circuit realizations, the universal active element CCII should be introduced by means of the hybrid three-port matrix

$$
V_{x}=V_{y}, \quad I_{y}=0, \quad I_{z}= \pm I_{x} .
$$

The orientations of all currents in this paper follow standard conventions, i.e. inwards the three-port. Several integrated circuits can be used as CCII where the sign in equalities (9) determines the type of the conveyor. The negative variant can be constructed by means of EL2082C while positive CCII is commercially available under the notation AD844.

The best way how to create an oscillator for modeling the dynamical behavior of the differential equation (1) is a parallel connection of some third-order admittance function with the shifted approximation of the $\mathrm{AV}$ characteristic of the ideal diode. Each active block $(\mathrm{CCII}+)$ in the oscillator is supposed to be in the linear regime of operation for all possible values of node voltages or branch currents. This proposition has the consequence while choosing impedance normalization factor.

\subsection{Nonlinear Function Synthesis}

The constant current source (CCS) includes one active block X5, resistor $R_{x}$ and external de voltage $V_{x}$ providing the output current $I_{\text {out }}=-I_{x}=-V_{x} / R_{x}$. It is obvious that the direction of the output current can be reversed and the 
value of resistor $R_{x}$ can be computed such that voltage $V_{x}$ can be taken as a positive or negative supply voltage.

CCS can be connected in parallel with a diode-resistor composite. Using such conception, only a single CCII+ is needed. The nonlinear device (diode) is strictly passive and can be replaced with other element with similar AV curve. Such modification will not make the qualitative changes in the global behavior of the circuit. The final circuit can be simpler, taking advantage of the active admittance network with reversed signs of all coefficients in (11). It turns out after applying the reasonable scaling factors that the real values of the circuit components (including $\mathrm{R}_{\mathrm{y}}$ ) make an effect of the forward-biased diode differential resistance negligible. Unfortunately, numerical analysis as well as the laboratory experiments reveal the problems with other non-ideal diode property, breakpoint. This voltage is typically located around $V_{p}=0.5 \mathrm{~V}$ (1N4007, BAT42). To overcome this obstacle, another CCII+ marked as X4 has been used as a voltage controlled current switch [6]. In this case the third-order admittance is passive.

\subsection{Individual Configurations}

Each final circuit is composed of a few basic building blocks which work simultaneously but their function can be understood separately. The main components are shown in Fig. 3 and ideally behave like a lossy grounded frequency dependent negative resistors (FDNR) with the possibility of the current inversion (this holds only for the first circuit). The associated admittance functions are

$$
\hat{Y}(\mathrm{~s})=I_{i n}(\mathrm{~s}) / V_{i n}(\mathrm{~s})= \pm\left[C_{i 1} C_{i 2} R_{i} \mathrm{~s}^{2}+\left(C_{i 1}+C_{i 2}\right) \mathrm{s}\right],(10)
$$

where $V_{\text {in }}(\mathrm{s})$ and $I_{\text {in }}(\mathrm{s})$ are Laplace transform of the input voltage and input current, respectively.

The first type of the final oscillator arises from (10) where one linear capacitor is replaced by another FDNR. Evidently there are a lot of possible combinations as shown in Fig. 5. Let assume that switching on/off the resistor $R_{y}$ must change the sign of the absolute coefficient of the characteristic polynomials as well as the real eigenvalue. For input voltage higher than zero the connection X4 and $\mathrm{D}$ behaves like a negative impedance converter. Due to this $G_{y}=-1 / R_{y}$ and the admittance $Y(\mathrm{~s})$ must consume the energy from the rest of the circuit. Taking into account the goal restriction $a_{2}=0$ we immediately get

$$
\begin{gathered}
Y(\mathrm{~s})=\tilde{Y}(\mathrm{~s})+\hat{Y}(\mathrm{~s}) \approx \widetilde{a}_{3} \mathrm{~s}^{3}+\tilde{a}_{2} \mathrm{~s}^{2}+\widetilde{a}_{1} \mathrm{~s}+\widetilde{a}_{0}- \\
-\hat{a}_{2} \mathrm{~s}^{2}-\hat{a}_{1} \mathrm{~s}=a_{3} \mathrm{~s}^{3}+a_{1} \mathrm{~s}+a_{0} .
\end{gathered}
$$

It means that another energy providing FDNR should be connected in parallel.

The second type of a third-order admittance function originated from the modified connection of two CCII+ used for the filter design and with the general impedances of all branches. The final equation for the input admittance is then simplified by substituting resistors, capacitors or even inductors and their serial or parallel interconnections as shown in Fig. 6.

The third type of the oscillator is based on the fundamental admittance cell shown in Fig. 4. The input admittance of such a cell is

$$
\hat{Y}(\mathrm{~s})=-\left[Y_{1}(\mathrm{~s})+Y_{3}(\mathrm{~s})+Y_{1}(\mathrm{~s}) Y_{3}(\mathrm{~s}) / Y_{2}(\mathrm{~s})\right] .
$$

As this equation suggests, any of the individual admittance can be frequency dependent as well as any of the used general impedance can be substituted by more complicated network leading to the desired linear part of the vector field. For example, the final structures illustrated in Fig. 7 can play this role. Only a single FDNR is used comparing to the first type of the oscillator.

Note that each circuit works in the hybrid voltagecurrent mode. State variables of the original mathematical model are measurable via their linear combinations.

In accordance to common form (11) the coefficients of the admittance function for the circuits in Fig. 5 are

$$
\begin{gathered}
a_{3}=c_{21} c_{22} c_{a} r_{2} r_{a}, a_{2}=c_{a} r_{a}\left(c_{21}+c_{22}\right)+c_{21} c_{22} r_{2}-c_{11} c_{12} r_{1}, \\
a_{1}=c_{21}+c_{22}-c_{a}-c_{11}-c_{22}, b_{0}=1 / r_{y}, a_{0}=1 / r_{b} .
\end{gathered}
$$

In these terms the normalized values of capacitors and resistors were used. Searching for concrete numbers is the problem from the area solving the system of the nonlinear algebraical equations. Such a task can cause the serious problems with finding the real positive values only. This is another advantage of the proposed structures of oscillators. Although the relative large amount of the circuit elements seems to be redundant it leads to the good overall results (quick convergence with zero error). It is not hard to learn that the satisfying performance also last for the second type of the circuit.
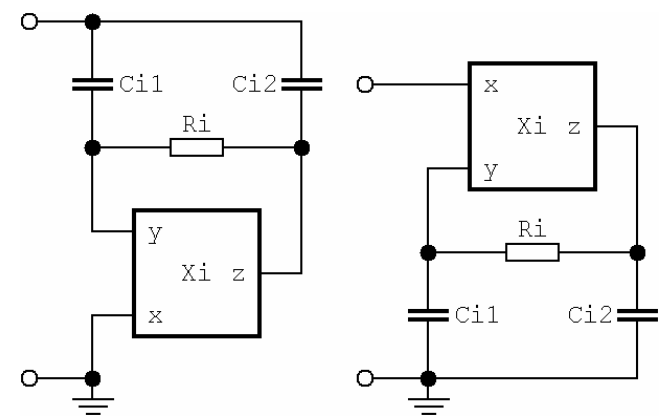

Fig. 3. Two reciprocal realizations of the FDNR using CCII+.

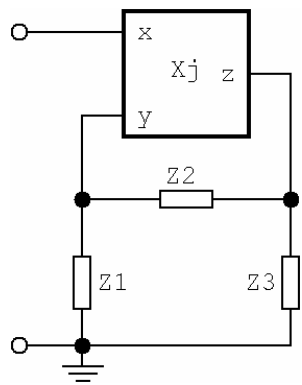

Fig. 4. CCII + based elemental admittance cell. 

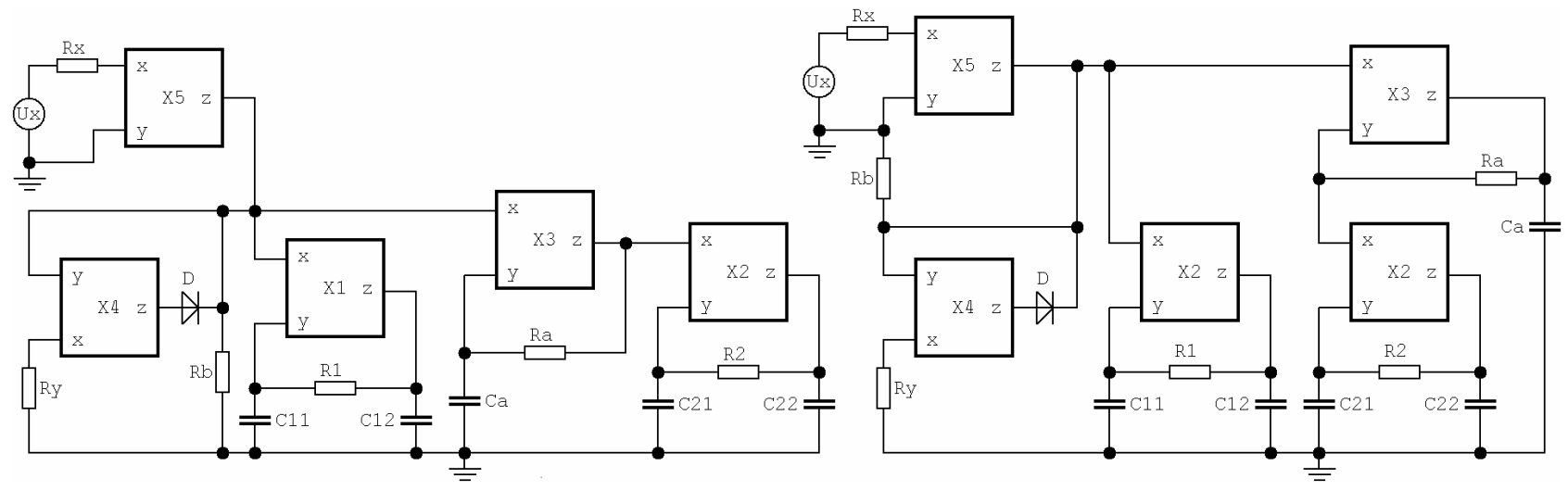

Fig. 5. Type I conservative chaotic oscillators.
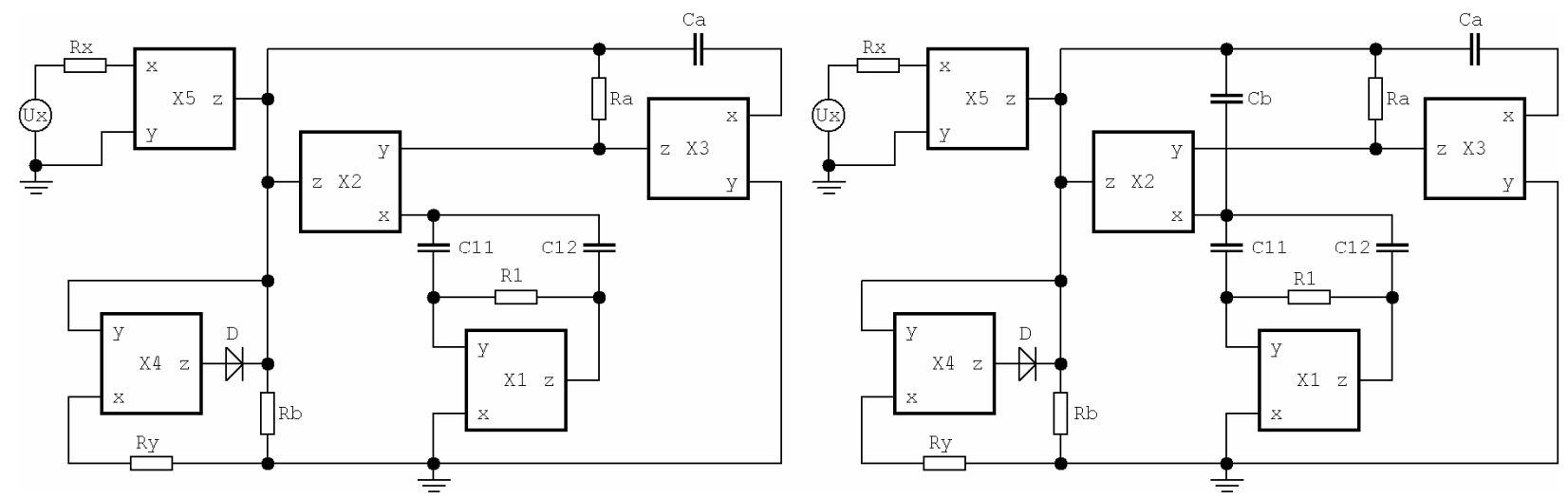

Fig. 6. Type II conservative chaotic oscillators.
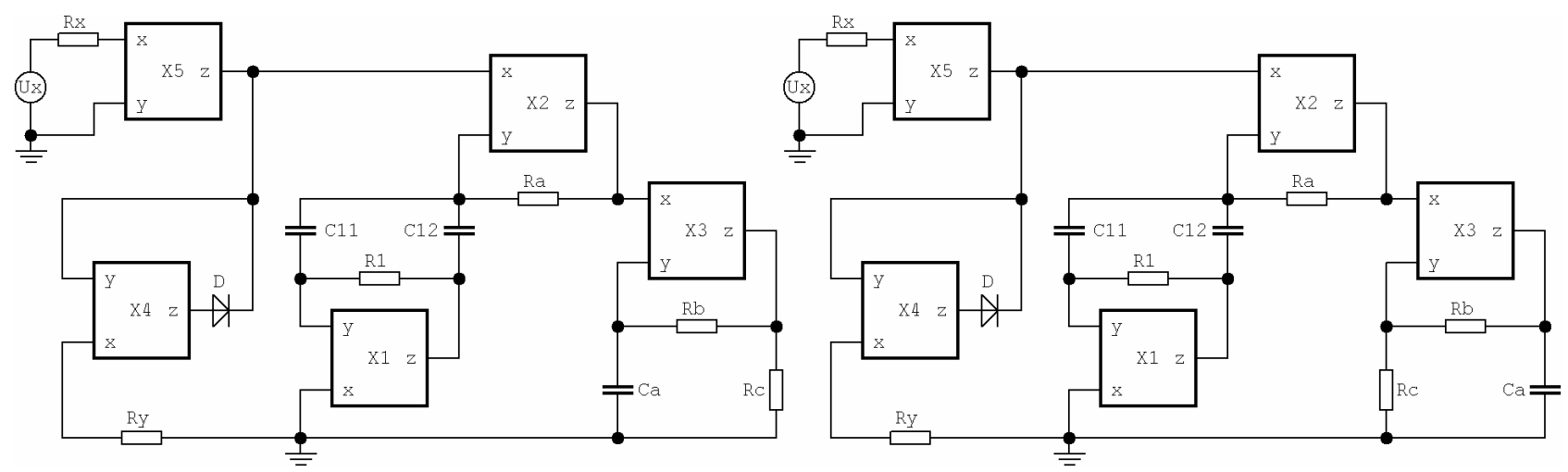

Fig. 7. Type III conservative chaotic oscillators.

Namely for the left circuit in Fig. 6 we get

$$
\begin{aligned}
& a_{3}=c_{11} c_{12} c_{a} r_{1} r_{a}, b_{0}=1 / r_{y}, a_{0}=1 / r_{b}, \\
& a_{2}=c_{a} r_{a}\left(c_{11}+c_{12}\right)-c_{11} c_{12} r_{1}, a_{1}=2 c_{a}-c_{11}-c_{12},
\end{aligned}
$$

and for the right circuit in Fig. 6 the coefficients are

$$
\begin{aligned}
& a_{3}=c_{11} c_{12} c_{a} r_{1} r_{a}, b_{0}=1 / r_{y}, a_{0}=1 / r_{b}, \\
& a_{2}=c_{a} r_{a}\left(c_{11}+c_{12}+2 c_{b}\right)-c_{11} c_{12} r_{1}, \\
& a_{1}=2 c_{a}-c_{11}-c_{12} .
\end{aligned}
$$

Note that slight modification of the circuit (adding a single capacitor) results into a change of the single equation. This feature can ensure convergence in many particular cases. The circuits shown in Fig. 7 differ only in the admittances of two branches. In this case this interchange does not affect the input admittance, which is in the form

$$
\begin{aligned}
& a_{3}=c_{11} c_{12} c_{a} r_{1} r_{a}\left(r_{b} / r_{c}+1\right), a_{0}=1 / r_{c}, b_{0}=1 / r_{y}, \\
& a_{2}=c_{a} r_{a}\left(c_{11}+c_{12}\right)\left(r_{b} / r_{c}+1\right)+c_{11} c_{12} r_{1}\left(r_{a} / r_{c}-1\right), \\
& a_{1}=\left(c_{11}+c_{12}\right)\left(r_{a} / r_{c}-1\right)+c_{a}\left(r_{b} / r_{c}+1\right) .
\end{aligned}
$$




\section{Experimental Results}

AV characteristic of the nonlinear two-port can be visualized by a current-sensing resistor $100 \Omega$ connected in series with a triangular wave generator. The result is shown in Fig. 8, where both input and output signals are centered. The concrete values of circuit components for the first type of configuration and (5) are $C_{11}=C_{12}=C_{21}=10 \mathrm{nF}$, $C_{22}=47 \mathrm{nF}, C_{a}=10 \mathrm{nF}, R_{1}=81 \mathrm{k} \Omega, R_{2}=15.5 \mathrm{k} \Omega, R_{a}=1.4 \mathrm{k} \Omega$, $R_{b}=10 \mathrm{k} \Omega$ and $R_{y}=5 \mathrm{k} \Omega$. For the same configuration and (6) some values change $C_{22}=100 \mathrm{nF}, R_{1}=177 \mathrm{k} \Omega, R_{2}=17 \mathrm{k} \Omega$, $R_{a}=600 \Omega, R_{b}=1.8 \mathrm{k} \Omega$ and $R_{y}=1.2 \mathrm{k} \Omega$. For the second type of oscillator with (5) the list of components is $C_{11}=C_{12}=10 \mathrm{nF}, C_{a}=24 \mathrm{nF}, R_{1}=14 \mathrm{k} \Omega, R_{a}=3 \mathrm{k} \Omega, R_{b}=10 \mathrm{k} \Omega$, and $R_{y}=5 \mathrm{k} \Omega$. Analogically, for the set (6) the following passive elements change $C_{a}=50 \mathrm{nF}, R_{a}=1.4 \mathrm{k} \Omega, R_{b}=1.8 \mathrm{k} \Omega$, and $R_{y}=1.2 \mathrm{k} \Omega$. Finally, the third type of oscillator with set (5) consists of $C_{11}=6 \mathrm{nF}, C_{12}=23 \mathrm{nF}, C_{a}=25 \mathrm{nF}$, $R_{1}=17.5 \mathrm{k} \Omega, R_{a}=800 \Omega, R_{b}=11 \mathrm{k} \Omega, R_{c}=10 \mathrm{k} \Omega, R_{y}=5 \mathrm{k} \Omega$, and for (6) we get $C_{11}=9 \mathrm{nF}, C_{12}=17 \mathrm{nF}, C_{a}=9 \mathrm{nF}$,
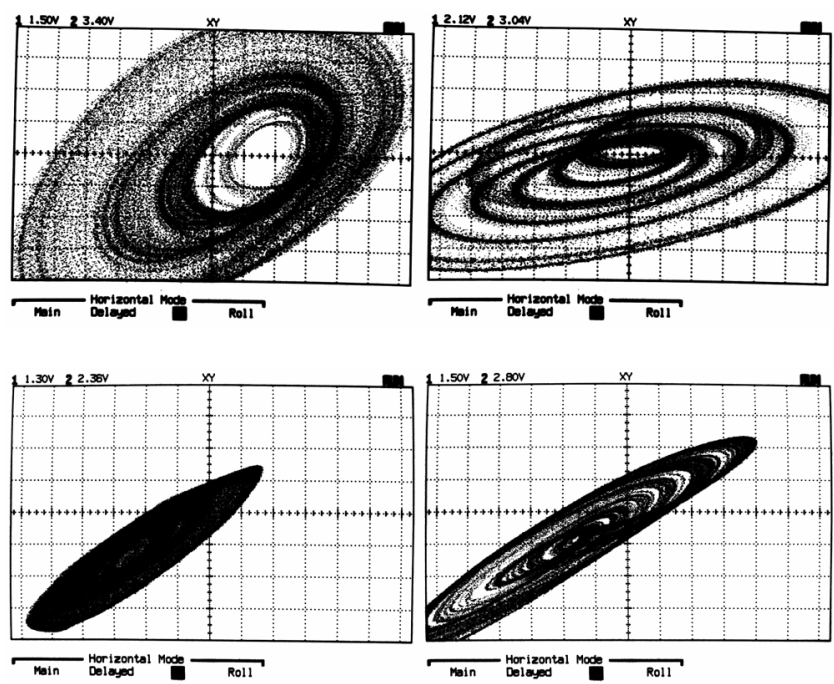

Fig. 9. Plane projection of chaotic attractors using XY display.

\section{Conclussion}

In this article several novel inductorless structures of the simple oscillators with associated volume conserving dynamics have been suggested and experimentally verified. The good agreement between theory and practice can be considered since the orbits closely related to conservative ones have been found. It is worth nothing that Pspice circuit simulator gives the same results. Moreover, single external dc source can be used to turn the oscillator inside a single-equilibrium regime or even into the equilibriumless one. The integrated circuit AD844 provides an extra node which acts as the output voltage follower. These buffered outputs allow us to observe other combinations of state variables without affecting the proper function of the oscillator.

The higher order admittances presented in this paper can be directly used for the generation of the multi-spiral
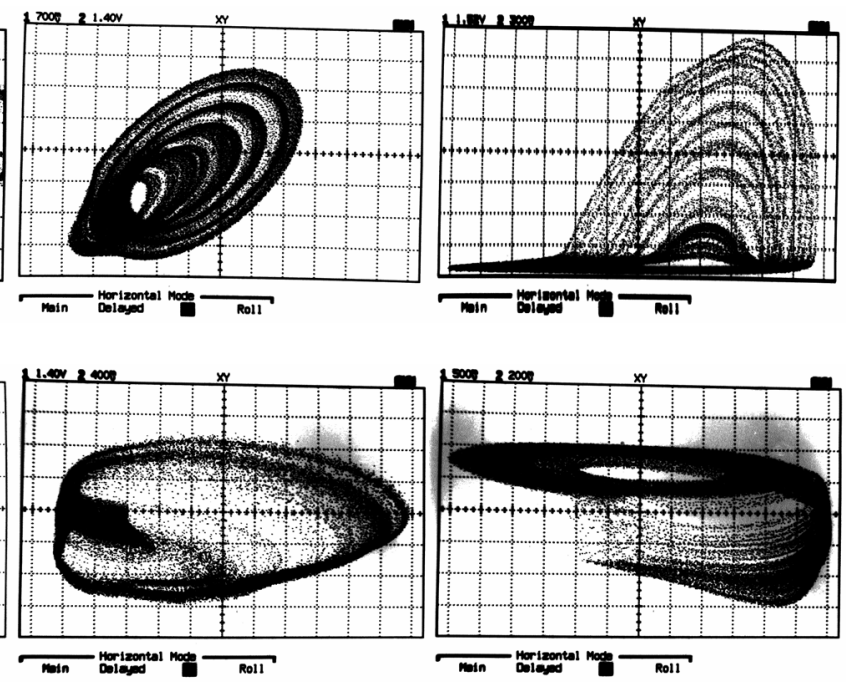

$R_{1}=14.7 \mathrm{k} \Omega, R_{a}=400 \Omega, R_{b}=19 \mathrm{k} \Omega, R_{c}=1.8 \mathrm{k} \Omega, R_{y}=1.2 \mathrm{k} \Omega$. Definitely, other sets of values are also possible for the different initial guess (Mathcad optimizer was used).

The digital oscilloscope HP54603B screenshots from a number of laboratory experiments are shown by means of Fig. 8 (AV characteristic of the nonlinear element and chaotic waveforms) and Fig. 9 (plane projections). Also the initial conditions close to zero should be introduced into the circuit before making each particular measurement.
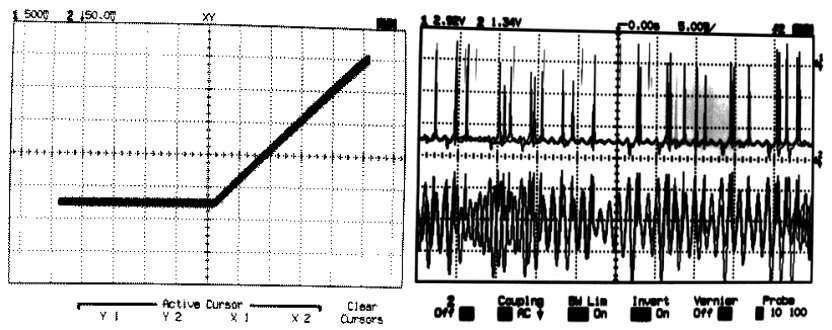

Fig. 8. Nonlinear AV characteristic and chaotic waveforms.

chaotic attractors. On the other hand, mathematical model of the studied systems is not suited for $2 \mathrm{D}$ or $3 \mathrm{D}$ grid attractors. More details about such oscillators can be found in some recent publications [7]. The symmetry of the vector field and the possibility of mapping absolute value onto the AV characteristic of the diode allow synthesized oscillators to model any shape of the state space attractors associated with two segment PWL vector field. The authors believe that much simpler oscillators with the same dynamics and employing only three CCII \pm can be discovered. This is the topic for further study.

\section{Acknowledgements}

The research described in this paper was financially supported by the research program MSM 0021630513. 


\section{References}

[1] SPROTT, J. C. Chaos and Time Series Analysis. Oxford University Press, 2003.

[2] GOTTLIEB, H. P. W., SPROTT, J. C., Simplest driven conservative chaotic oscillator. Physics Letters A, 2001, vol. 291, p. 385 - 388.

[3] PETRŽELA, J. Wideband signal generator based on chaos. In Proceedings of the $12^{\text {th }}$ Electrotechnical and Computer Conference ERK 2004. Portorož (Slovenia), 2004, p. 61 - 64.

[4] SPROTT, J. C., LINZ, S. J. Algebraically simple chaotic flows. International Journal of Chaos Theory and Applications, 2000, vol. 5, no. 2, p. 1 - 20.

[5] PETRŽELA, J., HANUS, S. On the optimization of the specific class of chaotic oscillators. In Proceedings of the $13^{\text {th }}$ Electrotechnical and Computer Conference ERK 2005. Portorož (Slovenia), 2005, p. 111 to 114 .

[6] LIU, S. I., WU, D. S., TSAO, H. W., WU, J., TSAY, J. H. Nonlinear circuit applications with current conveyors. In IEE Proceedings- $G$, 1993, vol. 140, no. 1, p. $1-6$.
[7] LU, J., CHEN, G. Generating multi-scroll chaotic attractors: theories, methods and applications. International Journal of Bifurcation and Chaos, 2006, vol. 16, no. 4, p. $775-858$.

\section{About Authors...}

Jiří PETRŽELA was born in Brno, Czech Republic, in 1978. His research interest is nonlinear dynamics, chaos theory and circuit design. He received the MSc. and PhD. degrees at the Brno University of Technology in 2003 and 2007 respectively. Now he is assistant professor at Department of Radio Electronics, Brno University of Technology.

Josef SLEZÁK was born in Zlín, Czech Republic, in 1982. His research interest is circuit theory, computer aided design of analog filters. He received the MSc. Degree at the Brno University of Technology in 2007. Now he is working towards a $\mathrm{PhD}$. degree at Department of Radio Electronics, Brno University of Technology.

\section{Call for Papers}

\section{Advanced Electronic Circuits}

\section{- from analog to digital $\bullet$ from low frequencies to millimeter waves}

\section{A Special Issue of Radioengineering}

\section{October 15, 2008: Submission of a paper}

A paper is requested being submitted via e-mail to editor@radioeng.cz. The manuscript has to be prepared in MS Word for Windows in Radioengineering Publishing Style. The example document with proper styles is available at:

\section{http://www.radioeng.cz}

Due to the very short publication time, extreme care has to be devoted to the formal processing of the paper. A paper of an inadequate form might be rejected from that reason. An author is encouraged to submit a PDF version of the paper together with the MS Word document in order to simplify handling the paper.

\section{October 31, 2008: Notification of acceptance}

Two independent reviewers review a paper submitted. Reviews are distributed to the authors via e-mail. If paper is accepted for publication, authors are asked to revise the paper in accordance with review comments.

\section{November 15, 2008: Submission of a revised paper}

The revised paper has to be delivered to the editors via e-mail in the form of the MS Word document as described in "Submission of a paper". 